\title{
Abnormalities of the Spatiotemporal Characteristics of Gait at the Onset of Freezing in Parkinson's Disease
}

\author{
Alice Nieuwboer, $\mathrm{PhD},{ }^{1} *$ René Dom, $\mathrm{MD},{ }^{2}$ Willy De Weerdt, $\mathrm{PhD},{ }^{1}$ Kaat Desloovere, $\mathrm{PhD},{ }^{3}$ \\ Steffen Fieuws, MSc, ${ }^{4}$ and Eva Broens-Kaucsik, $\mathrm{MSc}^{1}$ \\ ${ }^{1}$ Department of Rehabilitation Sciences of the Faculty of Physical Education and Physiotherapy, Katholieke Universiteit \\ Leuven, Belgium \\ ${ }^{2}$ Department of Neuroscience and Psychiatry, Katholieke Universiteit Leuven, Belgium \\ ${ }^{3}$ Department of the Faculty of Medicine, Gait Analysis Laboratory, Katholieke Universiteit Leuven, Belgium \\ ${ }^{4}$ Biostatistical Centre, Katholieke Universiteit Leuven, Belgium
}

\begin{abstract}
We investigated the spatiotemporal variables of gait leading up to freezing. Gait analysis was carried out on 14 patients with Parkinson's disease in the off phase of the medication cycle. A computerised, three-dimensional gait analysis system was used to measure the walking pattern. After several trials of normal walking with voluntary stopping, distracting manoeuvres and obstacles on the walkway were used to provoke freezing or festination. The gait variables of normal (off phase), festinating, prestop, and prefreezing strides were analysed using analysis of variance for repeated-measures. $\mathrm{Ca}$ dence was excessively increased $(68 \%)$ and stride length decreased $(69 \%)$ during festination compared with normal off
\end{abstract}

walking; a pattern which remained pronounced when comparing prefreezing strides with normal stopping. Analysing in more detail the three steps before a freeze, we found a progressive decrease of stride length and stable cadence rates and proportions of double support phases. The relationship between cadence and stride length exhibited an exponential increase of cadence with a decreasing stride length during festination and freezing. Results suggest that freezing is caused by a combination of an increasing inability to generate stride length superimposed on a dyscontrol of the cadence of walking. () 2001 Movement Disorder Society.

Key words: gait; Parkinson's disease; kinematics
Freezing is a common and disabling problem for people with Parkinson's disease (PD), especially in the advanced stages. Fifty-three percent of patients with a disease duration of more than 5 years experience regular freezing. ${ }^{1}$ The problem does not consistently respond to pharmacological intervention, a notion which has led to the distinction between on and off freezing. ${ }^{2}$ The clinical manifestation of freezing is unpredictable, not only dependent on the rise and fall of dopamine levels, but also on variations in sensory and cognitive input and on changes in motor task requirements. ${ }^{3,4}$ Freezing most frequently occurs when initiating gait $(85 \%)$, when turn-

*Correspondence to: Dr. Alice Nieuwboer, Department of Rehabilitation Sciences, Faculty of Physical Education and Physiotherapy, Tervuursevest 101, 3001 Heverlee, Belgium.

E-mail: alice.nieuwboer@flok.kuleuven.ac.be

Received 10 September 2000; Revised 30 January 2001; Accepted 5 March 2001

Published online 2 November 2001. ing $(45 \%)$, and when walking through a narrow passage or doorway $(25 \%) .^{2}$ A functional goal-directed task performed at the same time as walking was found to compromise gait performance generally and was observed to trigger freezing, suggesting an influence of attention on the problem. ${ }^{5}$

All these factors together make the study into the origins of freezing a challenging task and may contribute to the fact that few studies exist that examine the motor features of freezing and its associated phenomena. Few studies (i.e., Ueno et al. ${ }^{6}$ ) use gait analysis to describe the walking pattern in relation to freezing. In five patients with PD, Ueno and colleagues compared normal with "frozen" gait, probably referring to the typical festinating steps during or prior to a freeze. Stepping frequency was found to be increased in "frozen" gait, and was accompanied by an incomplete shift of the centre of pressure from one foot to the other. Three studies looked into the kinematics and kinetics of gait initiation in $\mathrm{PD}$, but no 
freezing was observed during testing. ${ }^{7-9}$ No specific abnormalities of the temporal and spatial patterns of gait initiation came to light other than that the variables were slower and smaller than in control subjects including execution of foot-off. We analyse the spatiotemporal characteristics of gait events preceding freezing. Spatiotemporal variables have been studied extensively in cued and uncued conditions during walking in PD. ${ }^{10-13}$ Slow walking velocity has been shown to reflect a difficulty in generating appropriate stride length, while cadence remained intact and was used as a compensatory mechanism. ${ }^{10,11}$ When manipulating stepping frequency with a metronome and measuring the corresponding stride length, the linear relationship between stride length and cadence was preserved in PD. ${ }^{14}$

The main purpose of the current study is to detect whether quantitative gait analysis yields specific features in the strides leading up to a freeze. We therefore compared the spatiotemporal stride features of (1) normal uninterrupted gait during the off phase; (2) a voluntary stop; and (3) freezing or festination. We hypothesised that the abnormalities which herald a freeze may be progressive, and so examined the differences between three consecutive strides prior to freezing. The stride lengthcadence relationship was also investigated across walking conditions, taking into account velocity, stride length, and cadence parameters.

\section{METHODS}

\section{Subjects}

Seventeen patients participated, of whom four were hospitalised at the time of study. Subjects were all diagnosed as having idiopathic Parkinson's disease according to accepted research criteria, ${ }^{15}$ had a recent history of regular freezing (with worsening during off), and were in a stable medical condition without acute neurological, orthopaedic, or cardiovascular conditions affecting gait. All subjects gave written informed consent prior to participation.

\section{Procedure}

Clinical data, including the Unified Parkinson's Disease Rating Scale (UPDRS) and the Hoehn \& Yahr scale, were collected in the on and off phases of the medication cycle.

Gait analysis took place in the gait laboratory of the University Hospital of the Katholieke Universiteit Leuven during the off phase. The hospitalised subjects were tested after the standard interval of at least 12 hours since the last intake of medication, ${ }^{16}$ omitting their normal morning dose. Patients who had to travel from home to the study were allowed to take their morning medication, omitting their mid-day dose. In these cases, we performed the gait tests after lunch as soon as a stable off period was reached as indicated by the patients themselves, using their normally experienced fluctuations as a point of reference. We defined the off period as the typical stable level of motor performance at the end of dose, when the action of medication is strongly decreased or absent. We defined wearing-off as the unstable period in which the patient is changing from on to off. A short clinical examination assessing tremor (UPDRS 20), rigidity (UPDRS 22) and distal tapping (UPDRS 23, 25, and 26) before and after gait analysis was used to check the stability of the off condition. Comparing the summed scores with a Wilcoxon signed rank test revealed no significant differences between the patients' condition before and after testing $\left(\overline{\mathrm{X}}_{\text {before }}=11.54 \pm 2.39 ; \overline{\mathrm{X}}_{\text {after }}=\right.$ $11.86 \pm 2.70 ; P=0.38$ ).

Patients walked without physical support on an 8-metre walkway. A physical therapist followed the subjects monitoring possible loss of balance and preventing falls. A six-camera system (Vicon 370; Advanced Mechanical Technology, Watertown, MA) registered gait performance at a resolution of $50 \mathrm{~Hz}$. Four normal video cameras augmented the set-up recording in the transversal, sagittal, and coronal plane. Two force plates (Advanced Mechanical Technology) were embedded in the walkway but were not used for data analysis. An integrated electromyographic (EMG) recording module registered activity in six muscle groups in both lower limbs (kinematic and EMG data to be presented ${ }^{17}$ ). Before gait analysis, lower limb dimensions, body height, and weight were registered to enable estimation of joint centre locations. Retroreflective markers were placed directly on the skin covering the anterior superior iliac spines, the lateral femur condyles, the lateral malleoli, the dorsal aspect of the foot between the second and third metatarsal heads, and on the calcaneus. Additional markers were attached approximately $5 \mathrm{~cm}$ away from the skin on plastic tubes attached to the lateral aspects of the thigh and shank and at the lumbosacral connection. A separate static trial using knee angle devices (KAD) was used to define the joint centres relative to the threedimensional planes.

The test procedure consisted of three parts in between which the patient rested for approximately 5 minutes. During the first part, patients were required to walk normally at a comfortable speed (normal condition). The order of the two subsequent parts was randomised to control for sequence effects. In the stop condition, patients were asked to walk normally and stop immediately at the oral cue provided by the investigator. The cue was given when patients hit the force plate with either the left 
or the right foot first. In the block condition, patients were exposed to freezing-provoking circumstances. Depending on the response of the patient, a single or a combination of two strategies was adopted to obtain freezing. The first strategy consisted of placing obstacles on the walkway to narrow it down. The obstacles were plastic tubes (height $80 \mathrm{~cm}$, width $10 \mathrm{~cm}$ ) or cubical shapes of approximately $60 \mathrm{~cm}^{2}$ made of plastic tubing to maximise its see-through effect and optimise the visibility of the markers. In case of a lack of a freezing response, we gave an additional distraction task which involved reciting multiplication tables, the days of the week, and months of the year in a reverse order (it has been established that carrying out a verbal cognitive task during walking markedly increases gait hypokinesia in $\mathrm{PD}^{5}$ ). Patients were required to walk through the narrow passage while repeating the verbal sequences aloud. Finally, in the continued absence of a freezing response, patients were asked to pass through a randomly placed obstacle course and in a second instance perform the earlier mentioned verbal distraction tasks as well. During these circumstances, patients would often freeze when having to make a turn or change direction. For data analysis, however, only freezes or festinations were considered which occurred without a directional change. Between 5 and 12 gait trials were conducted for both normal and stop conditions. For the block condition, patients carried out between 16 and 26 trials. All measurements were completed within 1 to 2 hours.

To obtain the desired freezing data, we tested five subjects on a second day within a period of 2 weeks. Time interval from the last medication, clinical status, and test conditions at the second test day were exactly the same as during the first test.

\section{Data Analysis}

A single score for the UPDRS items including multiple body parts was generated based on the worst function in case of asymmetry. We selected the valid gait laboratory trials for statistical analysis from the video recordings. During the block conditions, patients would either freeze or festinate when exposed to the freezingprovoking circumstances. Two trials out of each condition were selected. Freezing trials were defined as yielding a sudden episode of involuntary cessation of gait when passing or approaching an obstacle (narrow walkway or obstacle course with or without distraction). Festination trials were identified as sudden episodes of an involuntary increase of the stepping rate of gait while continuing to progress towards or through an obstacle course (not coming to a halt). To obtain representative data for normal walking, the three middle strides of three normal gait trials were used for analysis. For stop and block conditions, it was possible to consistently select two successful trials. The three middle strides of a festination interval were selected for statistical analysis. Before a freeze, the three consecutive strides closest to coming to a halt were chosen, excluding the final incomplete step. For the stop condition, the two or one complete strides occurring between the stop signal and the actual stop, as identified by the force plate recordings, were analysed. To warrant comparable block and stop data, we matched the trials according to the stance leg on which a freeze or stop would end (right or left).

Spatiotemporal profiles for both feet, including cadence expressed as the number of half cycles per minute, were calculated using Vicon Clinical Manager software (Oxford Metrics) using manual delineation of initial and terminal contact of the foot as markers for the gait cycles. The intertester reliability of this procedure was investigated by comparing the spatiotemporal data of normal and festinating strides of 10 patients analysed by two independent testers. We found that the intraclass correlation coefficients (ICC 1,2$)^{18}$ ranged from 0.93 to 1 , and 0.88 to 1 for the normal and festination conditions, respectively.

We analysed the consistency between strides and trials in every condition using an analysis of variance (ANOVA) repeated-measures procedure. No significant or systematic differences were found to justify the averaging of the normal, stop, and festination data. As one of the hypotheses of the study addressed the difference between the prefreezing strides, the data for each of these strides were averaged from two trials. Hereafter, the three strides preceding freezing will be indicated as

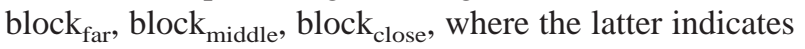
the stride closest to the block and so on, in reverse order. Missing values were generated because not every patient could provide all the required data due to the variable manifestation of the freezing and festination phenomena. After preliminary data analysis, we were left with representative stride parameters of six conditions: normal, stop, festination, block $\mathrm{far}_{\text {far }}$, block $\mathrm{middle}_{\text {e }}$, and block $_{\text {close }}$. An ANOVA for repeated measures was employed to analyse the strides between conditions. We compared normal gait with festination, block (freezing) with the voluntary stop condition and block with festination. To test the hypothesis of a possible accumulation of abnormality before a freeze, statistical comparison was carried out between the three block strides. The relation between stride length and cadence (dependent variable) was explored using a linear regression with an unstructured variance matrix to model the dependency between the six repeated measurements. We statistically verified the need for a qua- 
dratic term to adequately model cadence. To determine the importance of walking condition in the relationship, we added this factor to the regression model. The SAS procedure PROC MIXED (v. 6.12) ${ }^{19}$ was adopted in both analyses, which takes into account patients with one or more missing values. Data for the right and left were analysed separately.

\section{RESULTS}

Three of 17 patients failed to freeze or festinate during testing; therefore, the trials of 14 patients were withheld for analysis. Table 1 summarises the clinical data of the nine male and five female participants, including the UPDRS motor part (III) scores in the on and off period. Three patients did not freeze at all but showed festination only, while the opposite was apparent in three other subjects (Table 2). One patient was unable to perform a voluntary stop without festinating, so that no valid stop trials could be selected. Patients were tested 6 to 15 hours after taking the usual dose of medication (Table 2). Table 2 summarises the freezing-provoking circumstances that were successful in eliciting a freezing or festinating response for each patient. We obtained a freezing response in four patients by narrowing the walkway down and in three by placing random obstacles. Four subjects needed supplementary distraction (verbal tasks) to elicit freezing. One patient suggested a possible freezing strategy himself by having his partner walk in front of him as if to block the walkway.

\section{Normal Versus Festination}

Table 3 summarises the results of the ANOVA for repeated measures comparing the stride characteristics during off walking with those obtained during a spell of festination. Because highly similar results were found for both feet, we report the results for the left side only. Table 3 shows that mean gait velocity during festination was significantly slower than normal gait speed indicat-

TABLE 1. Clinical characteristics of patients

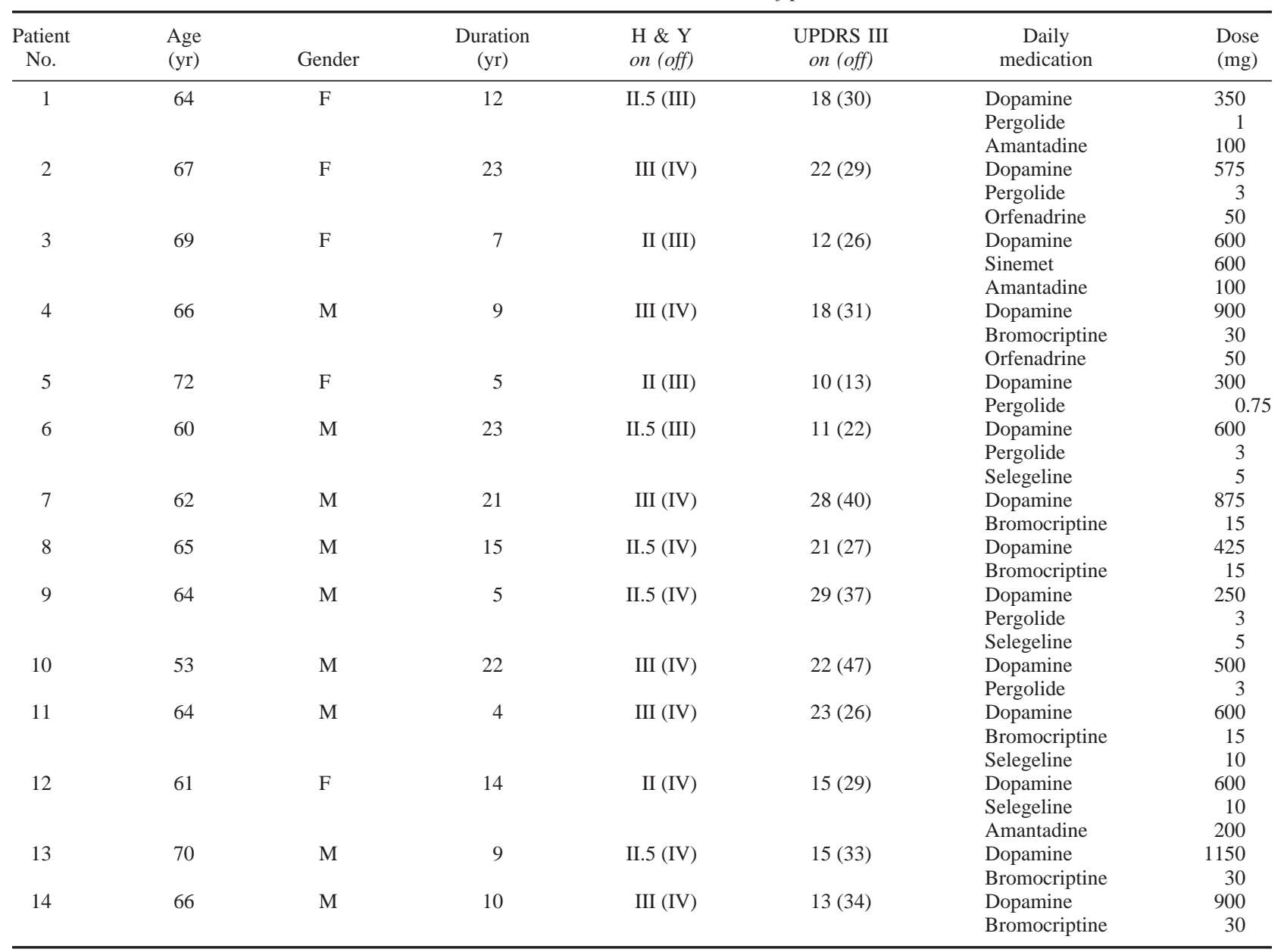

H \& Y, Hoehn \& Yahr; UPDRS, United Parkinson's Disease Rating Scale. 
TABLE 2. Strategies which provoked freezing or festination

\begin{tabular}{ccccl}
\hline $\begin{array}{c}\text { Patient } \\
\text { No. }\end{array}$ & $\begin{array}{c}\text { Number of } \\
\text { tests needed } \\
\text { to freeze }\end{array}$ & $\begin{array}{c}\text { Time } \\
\text { off (hr) }\end{array}$ & $\begin{array}{c}\text { Missing } \\
\text { data }\end{array}$ & Freezing-provoking strategy \\
\hline 1 & 2 & 6 & - & Random obstacles \\
2 & 2 & 13 & - & Random obstacles + distraction \\
3 & 1 & 15 & - & Narrowing + distraction \\
4 & 1 & 6 & - & Narrowing + distraction \\
5 & 1 & 7 & - & Random obstacles \\
6 & 1 & 6 & - & Narrowing \\
7 & 2 & 7 & S + B & Narrowing + distraction \\
8 & 1 & 6 & F & Narrowing \\
9 & 2 & 7 & B & Person in front \\
10 & 1 & 15 & F & Spontaneous \\
11 & 2 & 6 & B & Random obstacles \\
12 & 1 & 14 & - & Spontaneous \\
13 & 1 & 6 & F & Narrowing \\
14 & 1 & 7 & - & Narrowing \\
\hline
\end{tabular}

$\mathrm{S}$, stop condition; B, block condition; F, festination condition

ing a reduction of $49 \%$. Similarly, mean stride length was decreased by $69 \%$ of the normal mean value. Cadence, however, increased significantly to 167.2 steps per minute compared with the reference value of 99.46 steps per minute; a proportional increase of $68 \%$. Mean double support duration $(42.5 \%)$ in the normal condition was higher than ratios reported for healthy people of 65 years of age which varied between $30 \%$ and $35 \% .^{5}$ During festination, double support phase increased to $56.1 \%$ of the gait cycle, but this was not found to be statistically significant $(P=0.12)$. Figure 1 depicts the cadence and stride length values obtained in all six conditions. The figure illustrates that for normal walking, the variability was much greater for stride length than for cadence, as is confirmed by the coefficients of variation, which was $43 \%$ for stride length and $9 \%$ for cadence.

\section{Stop Versus Block}

Table 4 presents the differences between the strides before a voluntary stop and the three strides before a freeze (taken together). It shows that prior to freezing, patients walked at significantly slower speed (65\% stop versus block $\left.\mathrm{close}_{\text {e }}\right)$ and with decreased stride length $(76 \%$ stop versus block $_{\text {close }}$ ) than just before stopping. In the stop condition, both mean velocity and mean stride length were smaller than in normal walking in the off phase, probably indicating normal slowing down before stopping. Table 4 and Figure $1 \mathrm{~A}$ indicate that mean cadence was significantly higher when patients started to freeze compared with the strides leading up to a stop (51\% stop versus block $\left._{\text {close }}\right)$. Prior to a stop, cadence tended to be already higher (113 steps/minute) than during normal walking (99.5 steps/minute). Within the gait cycle, the mean proportion spent in the double support phase was significantly greater before freezing than be-
TABLE 3. Statistical analysis of spatiotemporal characteristics of normal and festinating strides for the left foot*

\begin{tabular}{lrcc}
\hline & $\begin{array}{c}\text { Normal } \\
\text { Mean (S.E.) }\end{array}$ & $\begin{array}{c}\text { Festination } \\
\text { Mean (S.E.) }\end{array}$ & $P$ \\
\hline Velocity (m/sec) & $0.57(0.07)$ & $0.29(0.06)$ & 0.0034 \\
Cadence (steps/minute) & $99.46(2.40)$ & $167.20(10.71)$ & 0.0001 \\
Stride length (m) & $0.67(0.08)$ & $0.21(0.05)$ & 0.0001 \\
Single support (\% GC) & $28.80(2.10)$ & $23.13(3.56)$ & NS \\
Double support (\% GC) & $42.55(4.31)$ & $56.06(7.29)$ & NS \\
\hline
\end{tabular}

*ANOVA analysis for repeated measures.

GC, gait cycle; NS, not signficant.

fore stopping and corresponding with this finding, time spent in single support was significantly shorter.

\section{Within-Block Comparison}

Repeated-measures ANOVA analysis partly confirmed the hypothesis that preblock events accumulate the closer one gets to a freeze. Table 4 and Figure 1B show that velocity and stride length progressively decreased to minimal proportions with statistically significant differences of $38 \%$ and $48 \%$ between block $_{\text {far }}$ and block $_{\text {close }}(P=0.04$ for velocity and $P=0.02$ for stride length). Although mean cadence values tended to increase when getting closer to the freeze with $16 \%$, this trend was not significant. The variability for cadence in the prefreezing strides was considerable (see Fig. 1A). Both single and double support proportions remained stable when approaching a freeze.

\section{Festination Versus Block}

No statistically significant differences were found between festination and block strides for all spatiotemporal variables. Figure 1A illustrates the strikingly similar cadence rates, particularly between the festination and block $_{\text {middle }}$ strides, suggesting that festination and freezing may be related phenomena. However, Figure 1B highlights that a wide variety of stride lengths were registered during festinating gait compared with a narrow band of minimal stride lengths in the block $_{\text {close }}$ strides, a difference which was almost significant $(P=0.08)$.

\section{Relation Between Stride and Cadence}

Figure 2 illustrates the left foot's stride-cadence relationship across the six conditions. The lines representing the individual patients show a variable pattern with a distinct common trend over the various conditions. From normal to stop, cadence rises gently when stride length decreases. This is followed by a steep upward inclination for cadence when stride becomes smaller and smaller during festination and block conditions. Statistically predicting cadence on the basis of stride showed that including a quadratic term was needed to model the asso- 


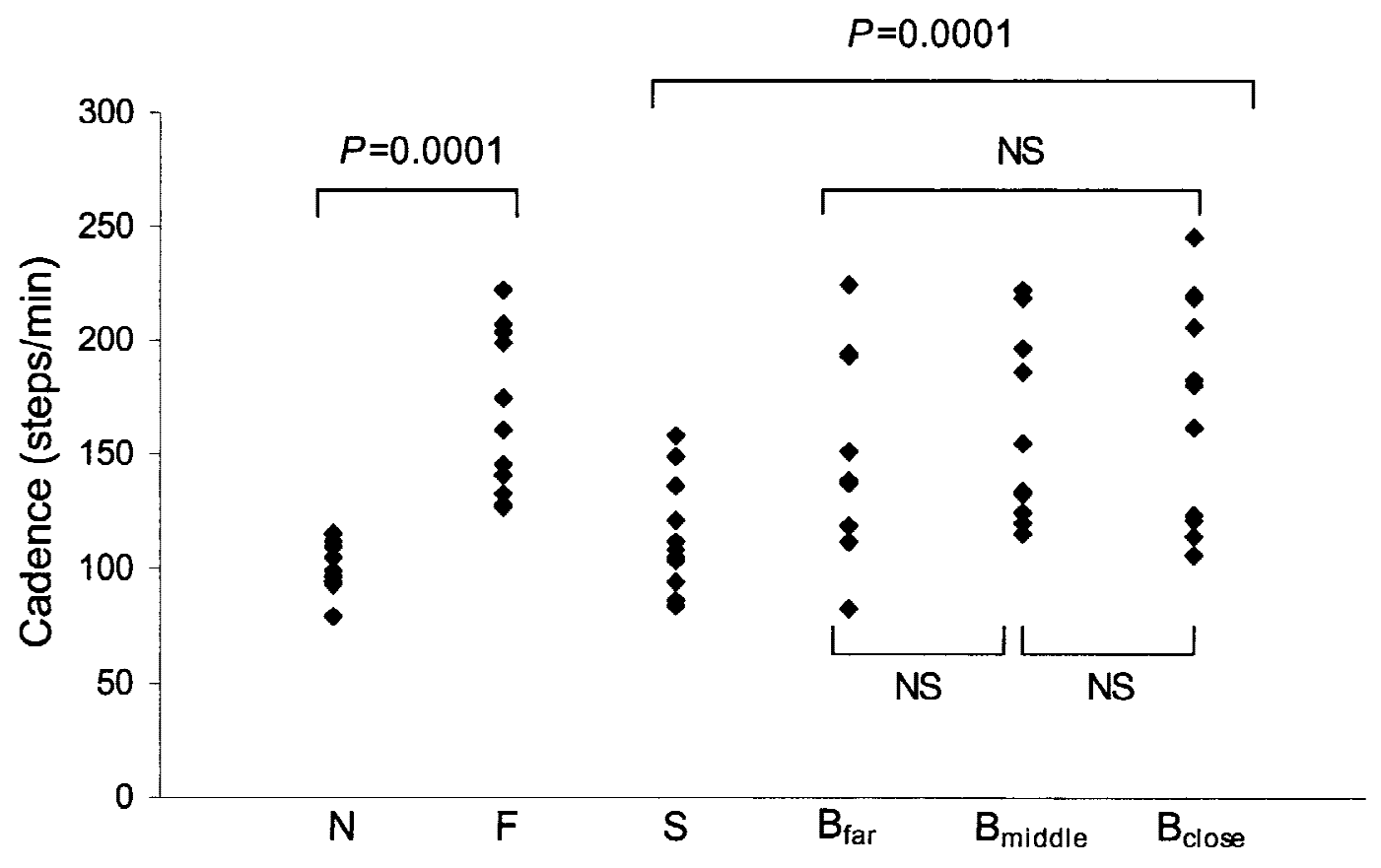

A

Condition

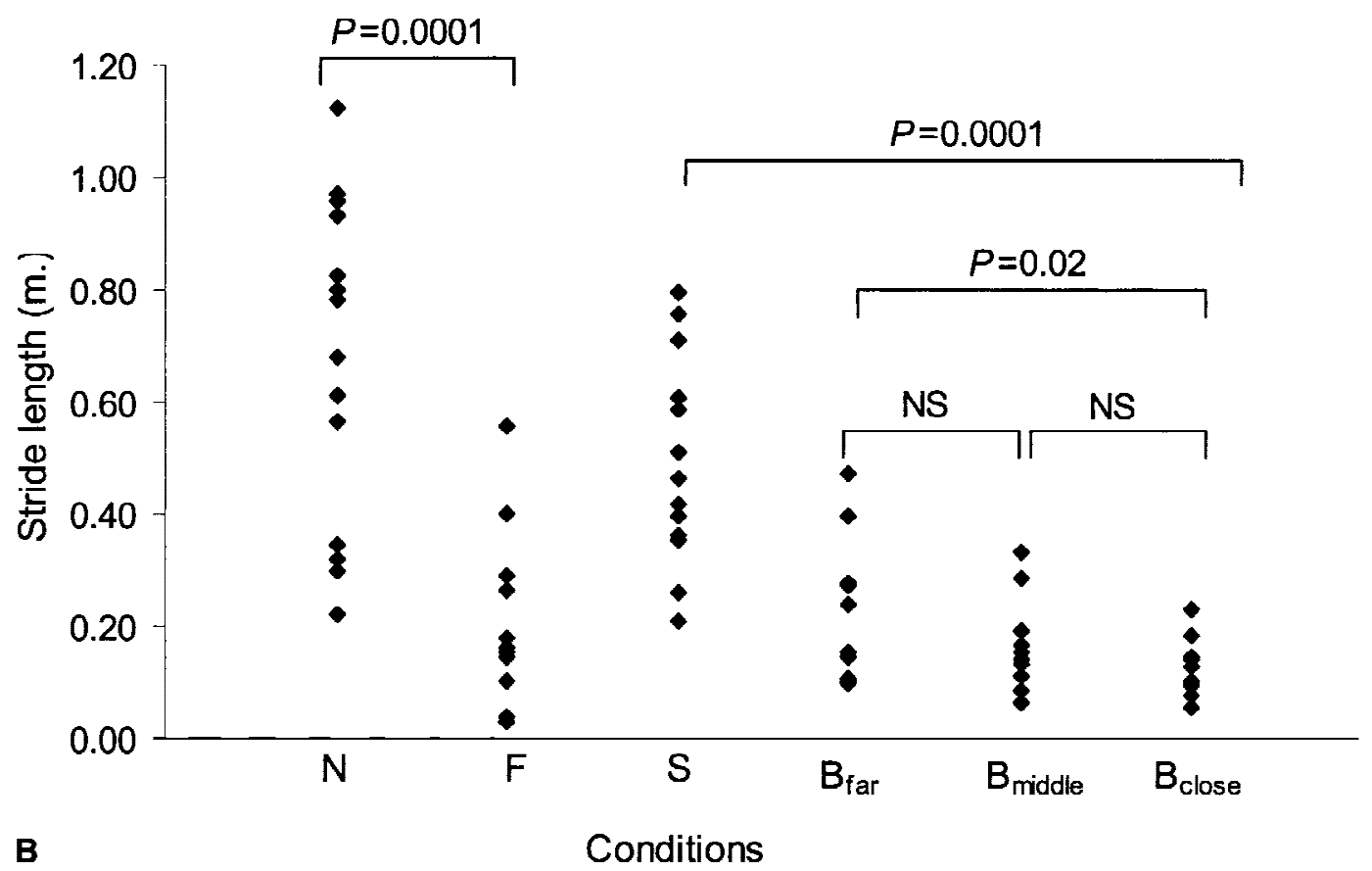

FIG. 1. A: Cadences of the normal (N), festination (F) stop (S) and block strides ( $\left.\mathrm{B}_{\text {far }}, \mathrm{B}_{\text {middle }}, \mathrm{B}_{\text {close }}\right)$ for all patients, left foot only $(\mathrm{n}=14)$. $P$-values pertain to the analyis of variance (ANOVA) for repeated measures. B: The stride lengths of the normal (N), festination (F) stop (S) and block strides $\left(\mathrm{B}_{\text {far }}, \mathrm{B}_{\text {middle }}, \mathrm{B}_{\text {close }}\right)$ for all patients, left foot only $(\mathrm{n}=14)$. $P$-values pertain to the ANOVA for repeated measures.

ciation, as both stride and stride squared were both highly significant predictors $(P=0.0001)$. We also found that this pattern was highly dependent on walking condition. Adding the factor "condition" to the predic- tion model revealed that the estimate for the quadratic term $(\beta=94.6$, S.E. $=15.9)$ reduced by $92.1 \%(\beta=$ $7.5, \mathrm{SE}=12.2)$ and proved no longer significant $(P=0.55)$. 
TABLE 4. Statistical analysis of spatiotemporal characteristics of strides taken before a voluntary stop and before a freeze for the left foot*

\begin{tabular}{|c|c|c|c|c|c|}
\hline & $\begin{array}{c}\text { Stop } \\
\text { Mean (S.E.) }\end{array}$ & $\begin{array}{c}\text { Block }_{\text {far }} \\
\text { Mean (S.E.) }\end{array}$ & $\begin{array}{l}\text { Block }_{\text {middle }} \\
\text { Mean (S.E.) }\end{array}$ & $\begin{array}{l}\text { Block }_{\text {close }} \\
\text { Mean (S.E.) }\end{array}$ & $P$ \\
\hline Velocity $(\mathrm{m} / \mathrm{sec})$ & $0.46(0.05)$ & $0.26(0.04)$ & $0.22(0.03)$ & $0.16(0.02)$ & 0.0001 \\
\hline Cadence (steps/minute) & $113.3(6.24)$ & $146.8(14)$ & $166(13.2)$ & $171(14.8)$ & 0.0001 \\
\hline Stride length $(\mathrm{m})$ & $0.5(0.05)$ & $0.23(0.04)$ & $0.16(0.02)$ & $0.12(0.01)$ & 0.0001 \\
\hline Single support (\% GC) & $31.53(1.69)$ & $23.48(3.61)$ & $22.25(2.91)$ & $23.1(3.73)$ & 0.0012 \\
\hline Double support (\% GC) & $41(3.52)$ & $56.78(6.11)$ & $59.24(5.49)$ & $61.4(6.07)$ & 0.0006 \\
\hline
\end{tabular}

ANOVA analysis for repeated measures.

* $P$-values represent the stop versus the 3 block strides taken together.

$\mathrm{GC}$, gait cycle.

\section{DISCUSSION}

Freezing proved a difficult problem to study in a laboratory setting. Despite the fact that patients were tested during the off phase of the medication cycle and were exposed to freezing-provoking circumstances, 3 of 17 patients failed to freeze and 5 needed retesting to do so. Previous studies have mentioned these difficulties. In two studies on gait initiation in PD, patients froze spontaneously in the corridor, in the doorway, on their way to the lab, and during turning but failed to freeze in the test situation. ${ }^{7,9}$ The difficulties to measure freezing may be explained by the state of heightened arousal and awareness of performance brought on by the test situation.

Different patients required different circumstances to obtain a freezing response, highlighting the variable and individual nature of the problem. In seven patients, the confrontation with visual stimuli suggesting a limited space or a change of direction produced freezing. In a

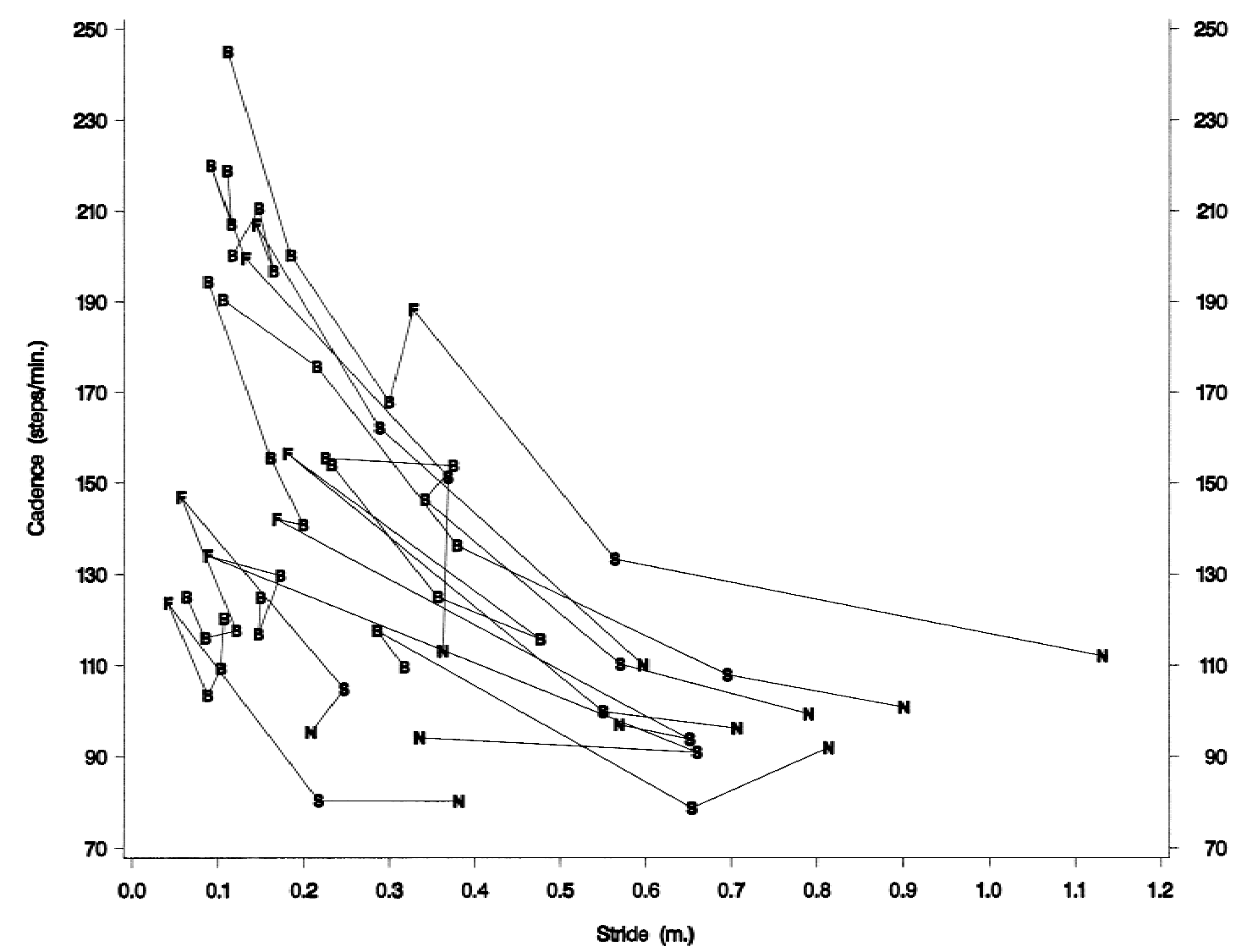

FIG. 2. Individual patient profiles $(\mathrm{n}=11)$ of the stride-cadence relationship in the normal $(\mathrm{N})$, stop ( $\mathrm{S})$, festination (F), and block (B) conditions, excluding patients who did not freeze. No distinction is made between $\mathrm{B}_{\text {far }}, \mathrm{B}_{\text {middle }}, \mathrm{B}_{\text {close }}$. 
study on the visual mechanisms involved in freezing, exposure to irrelevant visual stimuli in the peripheral visual field, especially the onset or offset of the stimuli, affected the time to initiate a manual task in PD but not in control subjects. ${ }^{20}$ The researchers postulated that abnormal interactions between substantia nigra, striatum, and the superior colliculi lead to an impairment of the orientating responses towards novel visual stimuli, making it harder to ignore irrelevant visual information. During both manual tasks and walking, images which fall in the peripheral visual field may divert the attention necessary to control gait and as a consequence produce a freeze. Four patients froze or festinated when having to perform an extra verbal task, confirming that attention is a crucial element in freezing. Earlier studies have shown that velocity and stride length also deteriorate during normal walking when a concurrent attention demanding task is being carried out. 5,10

We compared freezing and associated phenomena with normal gait during the off phase of the medication cycle. Walking in the off condition was already abnormal and varied strongly among patients. Mean gait velocity was $0.57 \mathrm{~m} / \mathrm{sec}( \pm 0.26)$, which is slower than the mean values found by other researchers during the on phase (mean, 0.83-1.15 m/sec). ${ }^{5,10}$ However, Blin and colleagues ${ }^{12}$ reported similar mean velocities both for on $(0.57 \pm 0.2 \mathrm{~m} / \mathrm{sec})$ and off phases $(0.47 \pm 0.2 \mathrm{~m} / \mathrm{sec})$. The present double support phase proportions (42.6\%) were prolonged, compared with previously reported values for the on $(29.6-35.9 \%)^{5,10,12}$ and off phases $(36.4 \%) .^{12}$

Against this background and regardless of the variable circumstances in which freezing episodes were provoked, a consistent pattern of results emerged from this study. We found an excessively increased cadence and decreased stride length comparing festination with normal (off) and prefreezing with prestopping gait. We demonstrated no significant differences between festinating and prefreezing strides, apart from a trend towards minimal mean stride length just before a freeze $(\bar{X}=0.12 \mathrm{~m})$ in contrast to festination $(\overline{\mathrm{X}}=0.21 \mathrm{~m}$.). These results confirm the findings of other studies suggesting that festination and freezing are closely linked phenomena. ${ }^{6,21}$ The difference between festination and actually coming to a standstill (as in freezing) seems to lie in the continued reduction of stride length before freezing while cadence remains stable. This suggests that the inability of generating amplitude over and above the production of excessive cadence leads to a breakdown of locomotion.

The overall scaling down of stride length is considered to be the main walking deficit caused by the deficient contribution of the basal ganglia to cortical motor set in PD. ${ }^{10,14}$ Results of animal studies imply that the basal ganglia may be involved in the internal cue production to string successive elements together in a movement sequence. $^{22,23}$ Failure to produce internal cues to integrate a sequence of gait cycles may cause an ever-diminishing stride length and cause freezing. Particularly, when patients are exposed to visual or attentional distraction and fall back on basal ganglia control, this faulty system may surface.

It is widely accepted that increased cadence serves as a compensatory mechanism for the fundamental decline of stride length in PD. ${ }^{10,13,24}$ When cadence is manipulated with a metronome, there is a positive linear relationship between stride length and cadence in both patients and controls until a break point at which stride length reached its maximum value. ${ }^{14}$ In the off phase, the break point cadence $(101.4 \pm 15.7$ steps/minute) was lower than during on (113.8 \pm 21.7 steps/minute) and lower than control group values (129.25 \pm 14.5 steps/ minute) ${ }^{14}$ In our study, mean cadence while festinating (167.19 steps/minute) or freezing (171 steps/minute) exceeded two standard deviations of the cadence, corresponding to previously reported ${ }^{14}$ control group break points. The disproportionately high cadences may be conceived as a hastening response reflecting a system out of control rather than a compensatory strategy. Rhythmic dyscontrol inherent to freezing and festination was also illustrated by the relationship between cadence and stride length requiring an additional quadratic term to describe the exponentially increased cadence with a declining stride length. A hastened footstep pattern may exaggerate the decreasing stride amplitude prior to freezing, not allowing enough time for sufficient movement generation. One of the features of bradykinesia is the failure to energise muscle activity up to the level necessary to complete a movement in the required amount of time. ${ }^{25}$ Also, force production during postural adjustment and weight shifting is ineffective and slower in $\mathrm{PD},{ }^{7}$ a deficit which may intensify during hastened stepping. As opposed to the reduced stride length, the elevated cadence rates did not significantly increase with every step nearer to a freeze. Possibly the maximum limits had already been reached, followed by a complete faltering of rhythm at the moment of freezing.

The issue of whether a fundamental deficit of movement timing is an intrinsic feature of PD is controversial. When patients were asked to step on the spot or walk to different metronome rhythms or music, they were able to closely match footstep timing to external auditory cues. $^{14,26}$ In contrast, hastening phenomena and motor blocks were reported for finger tapping ${ }^{27}$ and repetitive lip movements, ${ }^{28}$ exacerbated by the absence of external cues ${ }^{29,30}$ and by simultaneous task performance. ${ }^{28} \mathrm{~A}$ 
study on the ability to slow cadence down in early PD showed an irregular rhythm and timing of steps, affecting a subgroup of patients only. ${ }^{31}$ The deficient time-keeping function was attributed to a disturbance of central pattern generators in the spinal cord under the supraspinal control of the mesencephalic locomotor region, pedunculopontine nucleus, and the basal ganglia via cholinergic neurones. The fact that the rhythmic abnormalities concerned only a limited patient group led to the assumption that the same patients may be prone to developing freezing and start hesitation at a later stage in the disease. In agreement with these findings, Konczak and colleagues ${ }^{28}$ found that the timing of finger and lip movements was not generally impaired but rather affected specific patients. Ziv and associates ${ }^{27}$ demonstrated that already in early PD a relationship exists between the incidence of manual motor blocks and freezing.

Within the gait cycle, we found significantly increased double support phases before freezing compared with stopping, a finding which was less pronounced in festination. This result may indicate that prior to freezing, patients experience postural instability, which they compensate for by increasing the time in which both feet are in contact with the ground. Knuttson and Martensson ${ }^{32}$ observed that in severely disrupted festinating gait, the centre of gravity is ahead of foot support, characterising a propulsive walking pattern. It is imminent that this situation leads to loss of balance and falls. An alternative explanation for the increased proportion in double support is the fact that an incomplete shift of the centre of pressure takes place before freezing. ${ }^{6}$ Deficient force production, a slower displacement of the centre of mass forward, and a slower unloading of the swing limb were found in studies on gait initiation. ${ }^{7-9}$ Hence, the increase of double support before freezing may reflect an inability to adequately transfer weight as a preparation for stepping.

In conclusion, the stride characteristics of the onset of freezing and to a lesser degree of festination showed severe and cumulative loss of stride length on top of greatly accelerated cadence consistent with hastening. Double support phases were prolonged, possibly compensating for the postural instability or heralding the inability to prepare for stepping. The enhanced cadence suggests that freezing is associated with a disruption of the rhythmic control of gait as well as with a reducing ability to control amplitude. We believe that a vicious circle of these events results in a complete breakdown of locomotion, eventually causing freezing. Future study should clarify the exact interplay between the deficits of amplitude generation and timing of movement in the etiology of freezing. Furthermore, consideration of the muscle activation patterns (EMG) and biomechanical factors may provide insight into the origins of this complex phenomenon.

Acknowledgments: We thank the Research Council of the Katholieke Universiteit Leuven and the Fund of Scientific Research-Flanders for their financial support of this project.

\section{REFERENCES}

1. Giladi N, Treves TA, Korczyn AD, et al. Freezing of gait in patients with advanced Parkinson's disease (abstr). Mov Disord 2000;15 (suppl. 3):177.

2. Giladi N, McMahon D, Przedborski S, et al. Motor blocks in Parkinson's disease. Neurology 1992;42:333-339.

3. Fahn S. The freezing phenomenon in Parkinsonism. Adv Neurol 1995;67:53-63.

4. Lamberti P, Armenise S, Castaldo V, et al. Freezing gait in Parkinson's disease. Eur Neurol 1997;38:297-301.

5. Bond JM, Morris M. Goal-directed secondary tasks: their effect on gait in subjects with Parkinson disease. Arch Phys Med Rehabil 2000;81:110-116.

6. Ueno E, Yanagisawa N, Takami M. Gait disorders in Parkinsonism: a study with floor reaction forces and EMG. Adv Neurol 1993;60:414-418.

7. Burleigh-Jacobs A, Horak FB, Nutt JG, Obeso JA. Step initiation in Parkinson's disease: influence of levodopa and external sensory triggers. Mov Disord 1997;12:206-215.

8. Rosin R, Topka H, Dichgans J. Gait initiation in Parkinson's disease. Mov Disord 1997;12:682-690.

9. Halliday SE, Winter DA, Frank JS, Patla AE, Prince F. The initiation of gait in young, elderly, and Parkinson's disease subjects. Gait Posture 1998;8:8-14.

10. Morris ME, Iansek R, Matyas TA, Summers JJ. Stride length regulation in Parkinson's disease: normalization strategies and underlying mechanisms. Brain 1996;119:551-568.

11. Morris ME, Iansek R, Matyas TA, Summers JJ. Ability to modulate walking cadence remains intact in Parkinson's disease. J Neurol Neurosurg Psychiatry 1994;57:1532-1534.

12. Blin O, Ferrandez AM, Pailhous J, Serratrice G. Dopa-sensitive and Dopa-resistant gait parameters in Parkinson's disease. J Neurol Sci 1991;103:51-54.

13. Zijlstra W, Rutgers AWF, Van Weerden TW. Voluntary and involuntary adaptation of gait in Parkinson's disease. Gait Posture 1998;7:53-63.

14. Morris M, Iansek R, Matyas T, Summers J. Abnormalities in the stride length-cadence relation in Parkinsonian gait. Mov Disord 1998;13:61-69.

15. Ward CD, Gibb WR. Research diagnostic criteria for Parkinson's disease. Adv Neurol 1990;53:245-249.

16. Defer GL, Widner H, Marié RM, et al. Core assessment program for surgical interventional theapies in Parkinson's disease (CAPSIT-PD). Mov Disord 1999;17:572-584.

17. Nieuwboer A, De Weerdt W, Dom R, et al. EMG profiles of gait at the onset of freezing in Parkinson's disease (in preparation).

18. Shrout PE, Fleiss JL. Intraclass correlations: uses in assessing rater reliability. Psych Bull 1979;86:420-428.

19. Littell RC, Milliken GA, Stroup WW, Wolfinger RD. SAS system for mixed models. 1st ed. Cary: SAS Institute Inc; 1996.

20. McDowell S-A, Harris J. Irrelevant peripheral visual stimuli impair manual reaction times in Parkinson's disease. Vision Res 1997;37: 3549-3558.

21. Shabtai H, Rozenberg E, Shabtai E, Giladi N. Festinating gait in Parkinson's disease (abstr). Mov Disord 2000;15 (suppl. 3):177. 
22. Brotchie P, Iansek R, Horne M. A neural network model of neural activity in the monkey globus pallidus. Neurosci Lett 1991;131: 33-36.

23. Brotchie P, Iansek R, Horne MK. Motor function of the monkey globus pallidus. Brain 1991;114:1667-1683.

24. Morris ME, Iansek R, Matyas TA, Summers JJ. The pathogenesis of gait hypokinesia in Parkinson's disease. Brain 1994;117:11691181.

25. Hallett M. Clinical neurophysiology of akinesia. Rev Neurol (Paris) 1990;146:585-590.

26. McIntosh GC, Brown SH, Rice RR, Thaut MH. Rhythmic auditory-motor facilitation of gait patterns in patients with Parkinson's disease. J Neurol Neurosurg Psychiatry 1997;62:22-26.

27. Ziv I, Avraham M, Dabby R, Zoldan J, Djaldetti R, Melamed E. Early-occurrence of manual motor blocks in Parkinson's disease: a quantitative assessment. Act Neurol Scand 1999;99:106-111.

28. Konczak J, Ackermann H, Hertrich I, Spieker S, Dichgans J. Control of repetitive lip and finger movements in Parkinson's disease: influence of external timing signals and simultaneous execution on motor performance. Mov Disord 1997;12:665-676.

29. Freeman JS, Cody FWJ, Schady W. The influence of external timing cues upon the rhythm of voluntary movments in Parkinson's disease. J Neurol Neurosurg Psychiatry 1993;56:1078-1084.

30. Georgiou N, Iansek R, Bradshaw JL, Phillips JG, Mattingley JB, Bradshaw JA. An evaluation of the role of internal cues in the pathogenesis of parkinsonian hypokinesia. Brain 1993;116:15751587.

31. Ebersbach G, Heijmenberg M, Kinderman L, Trottenberg T, Wissel J, Poewe W. Interference of rhythmic constraint on gait in healthy subjects and patients with early Parkinson's disease: evidence for impaired locomotor pattern generation in early Parkinson's disease. Mov Disord 1999;14:619-625.

32. Knutsson E, Martensson A. Posture and gait in parkinsonian patients. In: Bles W, Brandt RH, editors. Disorders of posture and gait. Amsterdam: Elsevier; 1986. p 217-229. 